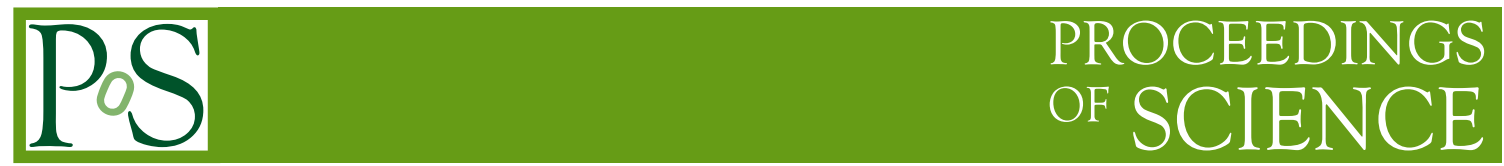

\title{
Early detections of properties of Gamma-Ray Bursts
}

\author{
G. Pizzichini* \\ INAF - IASF Bologna \\ E-mail: pizzichiniliasfbo.inaf.it
}

Some of the Gamma-Ray Burst properties which were later much better proved were, in some cases, already reasonably evident in the first detections: in particular the X-ray afterglow, the bimodal duration and, possibly, also the optical emission.

Swift: 10 Years of Discovery,

2-5 December 2014

La Sapienza University, Rome, Italy

* Speaker. 


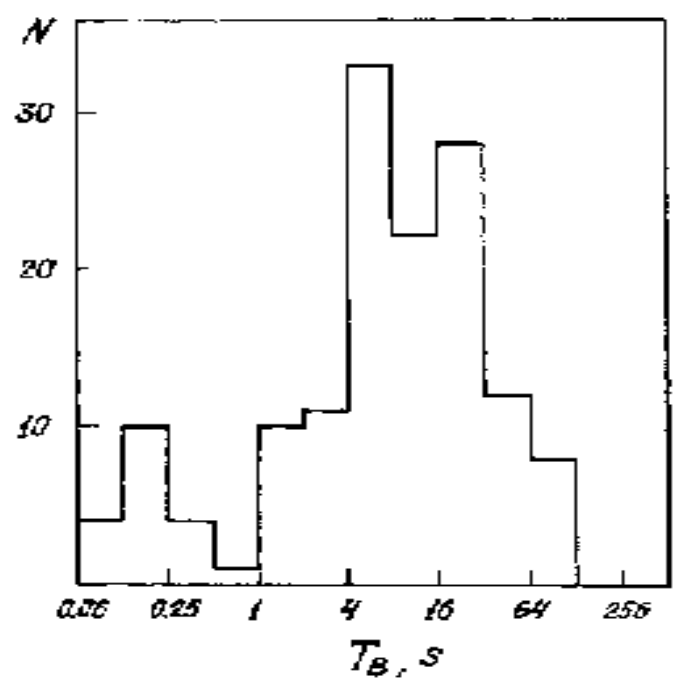

Figure 1: Histogram of GRB durations from the Konus catalog [2]

\section{Bimodal duration}

Cline and Desai [1] already suggested a different class of very short Gamma-Ray Bursts (GRBs). Mazets et al. [2] show in their I and II Konus catalog a clustering of GRB durations at about 2s. The bimodal distribution of GRB durations was later proved with much better statistics by Kouveliotou et al. [3]. in the BATSE catalog.

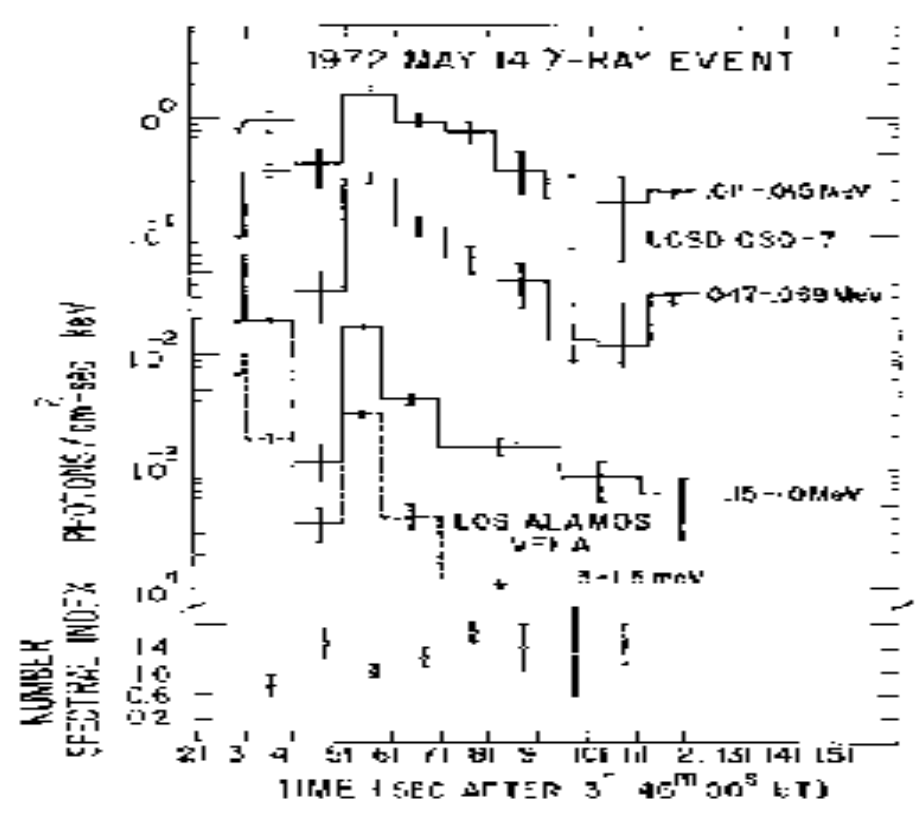

Figure 2: Detection of GRB720514 by [4] 


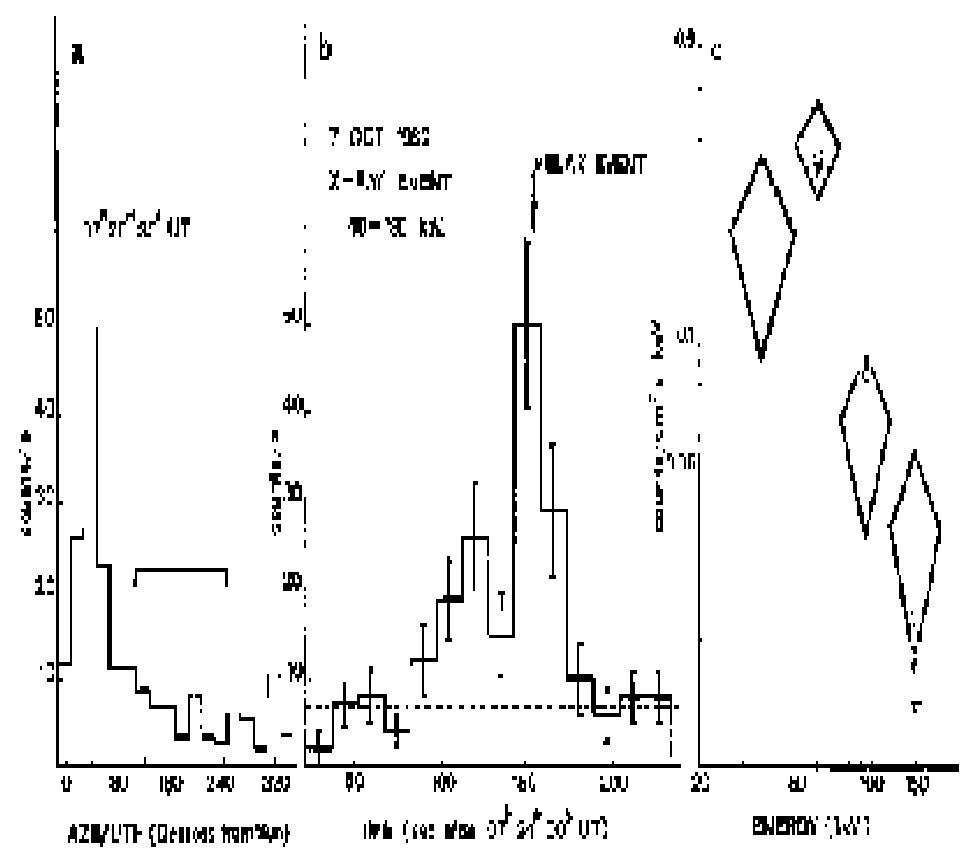

Figure 3: Detection of GRB691007 by [5]

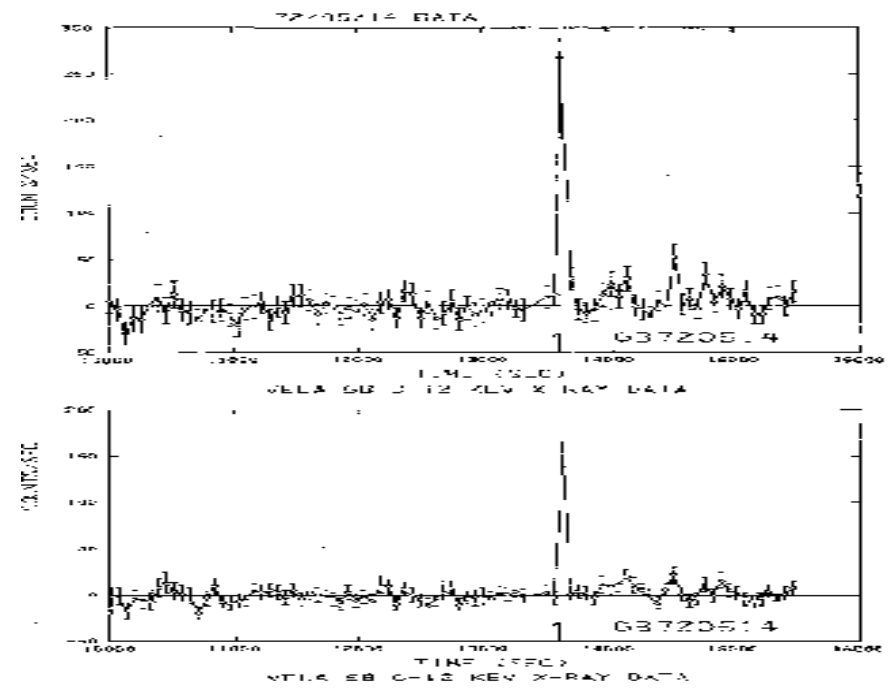

Figure 4: Detection of GRB720514 by [7]

\section{X-ray afterglow}

X-rays down to $10 \mathrm{keV}$ were already detected by Wheaton et al. [4] from GRB720514. Palumbo, Pizzichini and Vespignani [5] detected 49-189 keV hard X-rays from GRB691007 for a much longer duration than the one reported by Klebesadel, Strong and Olsen [6]. Terrell et al. [7] detected 3-12 keV photons from GRB720514 and GRB740723, also probably for a much longer duration than the Vela detections. In all three cases X-ray error boxes, although quite large, were 
obtained.

\section{Optical emission}

Schaefer et al., [8] encouraged the search for optical counterparts of GRBs by showing "Two probable optical flashes from Gamma-Ray Bursters" (GRB791105 and GRB790113) on archival photographic plates, taken however at distant times from the two GRBs. Then Van Paradijs et al. [9] detected the contemporaneous OT from GRB970228.

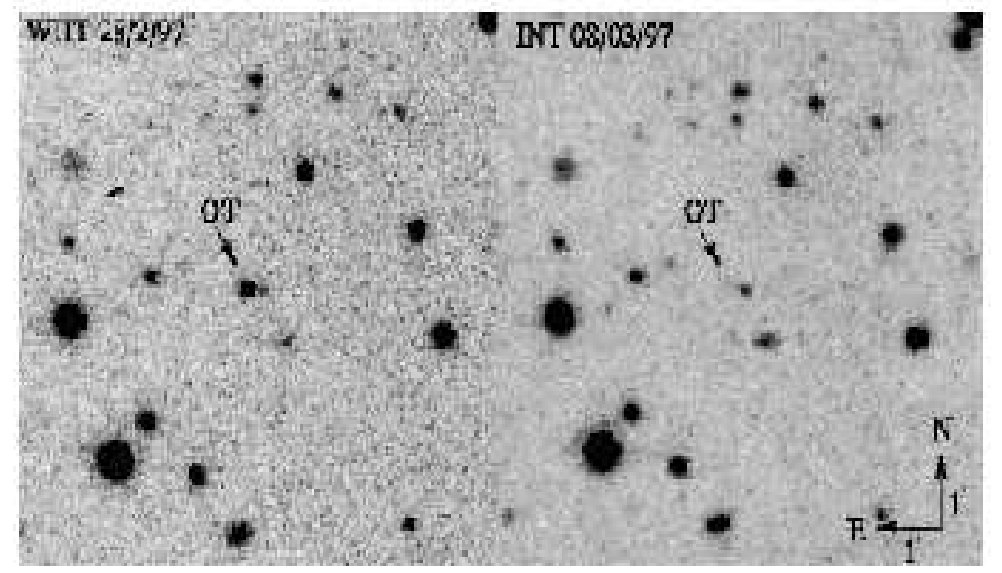

Figure 5: Detection of the OT from GRB970228 by [9]

\section{References}

[1] T. L. Cline and U. D. Desai, Progress in gamma ray burst astronomy, Proceedings 9th ESLAB Symposium: ESRO, The context and status of gamma ray astronomy (1974) 37

[2] E. P. Mazets et al., Catalogue of cosmic gamma-ray bursts from the Konus experiment data, ApSS80 (1981) 3

[3] C. Kouveliotou et al., Identification of two classes of gamma-ray bursts, Apj413 (1993) L101

[4] Wm. A. Wheaton et al., The Direction and Spectral Variability of a Cosmic Gamma-Ray Burst, ApJ185 (1973) L57

[5] G. G. C. Palumbo, G. Pizzichini and G. R. Vespignani, Observation of a celestial X-ray burst in coincidence with a gamma-ray burst,ApJ 189 (1974) L9

[6] R. W. Klebesadel, I. B. Strong and R. A. Olson, Observations of Gamma-Ray Bursts of Cosmic Origin,ApJ 182 (1973) L85

[7] J. Terrell et al., Observations of two gamma-ray bursts by VELA X-ray detectors, ApJ 254 (1982) 279

[8] B. E. Schaefer et al., Two probable optical flashes from gamma-ray bursters, ApJ 286(1984) L1

[9] J. van Paradijs et al., Transient optical emission from the error box of the gamma-ray burst of 28 February 1997, Nature 386 (1997) 686 